S. Breulmann and W. Kohnen

Nagoya Math. J.

Vol. 155 (1999), 153-160

\title{
TWISTED MAAß-KOECHER SERIES AND SPINOR ZETA FUNCTIONS
}

\author{
STEFAN BREULMANN AND WINFRIED KOHNEN
}

\begin{abstract}
It is shown that a Siegel-Hecke eigenform of integral weight $k$ and genus 2 is uniquely determined by its Fourier coefficients indexed by $n T$ where $T$ runs over all half-integral positive definite primitive matrices of size 2 and $n$ over all squarefree positive integers. The proof uses analytic arguments involving Koecher-Maaß series and spinor zeta functions.
\end{abstract}

\section{$\S 1$. Introduction}

A classical result of Hecke can be stated as follows: Let $f$ and $g$ be elliptic cuspidal Hecke eigenforms of the same weight with Fourier coefficients $a(n)$ resp. $b(n)(n \in \mathbb{N})$. Assume that $a(1)=b(1)$ and that $a(p)=b(p)$ for every prime $p$. Then $f=g$.

Our aim in this paper is to give an analogous result in the case of modular forms of degree two. Let $S_{k}\left(\Gamma_{2}\right)$ be the complex vector space of Siegel cusp forms of weight $k \in \mathbb{Z}$ for the Siegel modular group $\Gamma_{2}:=$ $\operatorname{Sp}(2 ; \mathbb{Z})$ of degree two. For $F \in S_{k}\left(\Gamma_{2}\right)$ we let $a(T)$ (T half-integral, positive definite) be the Fourier coefficients of $F$. We will show

Theorem. Let $F, G \in S_{k}\left(\Gamma_{2}\right)$ be Hecke eigenforms with Fourier coefficients a $(T)$ resp. $b(T)$. If $a(n T)=b(n T)$ for all half-integral, positive definite, primitive matrices $T$ and all square-free integers $n \in \mathbb{N}$, then $F=G$.

To prove the theorem we proceed as follows.

According to $[\mathrm{An}]$ there is an identity relating the Maaß-Koecher series of a Hecke eigenform $F$ with the spinor zeta function of $F$. Twisting this identity by Groessen characters and using the functional equations both for the twisted Maaß-Koecher series and for the spinor zeta functions, we obtain after some calculations that the twisted Maaß-Koecher series of $F$ and $G$ must coincide. (Note that a similar idea already has been used in $[\mathrm{Ko}]$ in the context of elliptic modular forms of half-integral weight.) By the converse theorem of Imai $([\operatorname{Im}])$ we therefore get $F=G$.

Received March 10, 1998. 
Acknowledgements. The authors would like to thank the referee for a useful remark.

\section{$\S 2$. Maaß-Koecher series}

If $F \in S_{k}\left(\Gamma_{2}\right)$ ( $k$ even) with Fourier coefficients $a(T)$ we denote by

$$
D_{F}(s):=\sum_{T>0 / \sim} \frac{a(T)}{\epsilon(T)(\operatorname{det} T)^{s}} \quad(\operatorname{Re} s \gg 0)
$$

the Maaß-Koecher series of $F$ (summation over a complete system of representatives of $\mathrm{GL}(2 ; \mathbb{Z})$-equivalence classes of half-integral, positive definite matrices $\left.T ; \epsilon(T):=\sharp\left\{U \in \operatorname{GL}(2 ; \mathbb{Z}): U^{t} T U=T\right\}\right)$. In the following we will use these series twisted by Groessen characters and for this we have to recall the Roelcke-Selberg spectral decomposition (cf. [Ku], [Im]).

Let $\mathbb{H}:=\{z \in \mathbb{C}: \operatorname{Im} z>0\}$ be the upper half plane and $\Delta$ the Laplace operator on $\mathbb{H}$. Denote by $\langle\cdot, \cdot\rangle$ the Petersson scalar product on the space $\mathcal{L}^{2}\left(\Gamma_{1} \backslash \mathbb{H}\right)$ where $\Gamma_{1}$ is the elliptic modular group acting on $\mathbb{H}$ in the usual way.

Let $v_{0}=\sqrt{\frac{3}{\pi}}$ and $\left\{v_{j}: j \in \mathbb{N}\right\}$ be an orthonormal basis consisting of cuspidal eigenfunctions for $\Delta$. Let $\Gamma_{1, \infty}:=\left\{\left(\begin{array}{cc}1 & * \\ 0 & 1\end{array}\right) \in \Gamma_{1}\right\}$ and let

$$
E_{u}(z):=\frac{1}{2} \sum_{\gamma \in \Gamma_{1, \infty} \backslash \Gamma_{1}}(\operatorname{Im} \gamma z)^{u} \quad(\operatorname{Re} u>1, z \in \mathbb{H})
$$

be the non-holomorphic Eisenstein series. We denote the analytic continuation of $E_{u}(z)$ to a meromorphic function on $\mathbb{C}$ with respect to the variable $u$ again by $E_{u}(z)$.

For any $f \in \mathcal{L}^{2}\left(\Gamma_{1} \backslash \mathbb{H}\right)$ the Roelcke-Selberg spectral decomposition is given by

$$
f(z)=\sum_{j=0}^{\infty}<f, v_{j}>v_{j}(z)+\frac{1}{4 \pi i} \int_{\operatorname{Re} \mathrm{u}=\frac{1}{2}}<f, E_{u}>E_{u}(z) d u \quad(z \in \mathbb{H}) .
$$

For $F \in S_{k}\left(\Gamma_{2}\right)$ let

$$
F_{t}(W):=F(i \sqrt{t} W) \quad(W>0, \operatorname{det} W=1 ; t \in \mathbb{R}, t>0) .
$$


For Re $s \gg 0$ we consider the Mellin transformation

$$
\widetilde{F}_{s}(W):=\int_{0}^{\infty} F_{t}(W) t^{s-1} d t
$$

As in [Im], p. 917 we identify the set of all positive definite matrices $W$ of determinant 1 with $\mathbb{H}$ so that we easily see that $\widetilde{F}_{s} \in \mathcal{L}^{2}\left(\Gamma_{1} \backslash \mathbb{H}\right)$.

Define

$$
\Lambda_{k}:= \begin{cases}\left\{u \in \mathbb{C}: \operatorname{Re} u=\frac{1}{2}\right\} \cup\left\{j \in \mathbb{N}_{0}: v_{j} \text { even }\right\}, & \text { if } k \text { is even, } \\ \left\{j \in \mathbb{N}_{0}: v_{j} \text { odd }\right\}, & \text { if } k \text { is odd. }\end{cases}
$$

For $\lambda \in \Lambda_{k}$ we let $\varphi_{\lambda}=\overline{\nu_{j}}$ or $\varphi_{\lambda}=E_{\bar{u}}$, respectively depending on whether $\lambda \in \mathbb{N}_{0}$ or not and define

$$
D_{F, \lambda}(s):=\sum_{T>0 / \sim} \frac{a(T) \varphi_{\lambda}\left(\frac{1}{\sqrt{\operatorname{det} T}} T\right)}{\epsilon(T)(\operatorname{det} T)^{s}} \quad(\operatorname{Re} s \gg 0),
$$

i.e. for $k$ even $D_{F, \lambda}(s)$ denotes the Maaß-Koecher series twisted by $\varphi_{\lambda}$. (Note that the constant implied in "》" can be chosen independently of $\lambda \in \Lambda_{k}$, cf. [Im], p. 932 ff.).

The following result is implicitly contained in [Im], however, for the reader's convenience we recall the proof.

LEMMA 2.1. Let $F \in S_{k}\left(\Gamma_{2}\right)$. If $F \neq 0$, then there exists $\lambda \in \Lambda_{k}$ such that $D_{F, \lambda}(s)$ does not vanish identically.

Proof. For Re $s \gg 0$ set $\Phi_{F, j}(s):=<\widetilde{F}_{s}, v_{j}>\left(j \in \mathbb{N}_{0}\right) \operatorname{resp} . \Phi_{F, u}(s):=$ $<\widetilde{F}_{s}, E_{u}>\left(u \in \mathbb{C}\right.$, Re $\left.u=\frac{1}{2}\right)$. The Roelcke-Selberg spectral decomposition for $\widetilde{F}_{s}$ says

$$
\widetilde{F}_{s}(z)=\sum_{j=0}^{\infty} \Phi_{F, j}(s) v_{j}(z)+\frac{1}{4 \pi i} \int_{\operatorname{Re} \mathrm{u}=\frac{1}{2}} \Phi_{F, u}(s) E_{u}(z) d u .
$$

Since $\widetilde{F}_{s}(z)$ is an even resp. odd function in $x=$ Re $z$ depending on whether $k$ is even or odd, in the sum only values $\lambda \in \Lambda_{k}$ can occur, and the integral is zero if $k$ is odd. 
Now assume that $\Phi_{F, \lambda}(s)=0$ for Re $s \gg 0$ and every $\lambda \in \Lambda_{k}$. Then $\widetilde{F}_{s}(z)=0$ for $z \in \mathbb{H}$ and Re $s \gg 0$. Hence by Mellin inversion we find that $F_{t}(W)=0$ for all $t$ and $W$ so that $F(i Y)=0$ for any $Y>0$. This implies $F=0$ in contrast to the assumption. Hence we can find $\lambda \in \Lambda_{k}$ such that $\Phi_{F, \lambda}(s)$ does not vanish identically.

If $\varphi_{\lambda}=\overline{v_{j}}\left(j \in \mathbb{N}_{0}\right)$ then by [Im], p. 927 we have for Re $s \gg 0$

$$
\Phi_{F, j}(s)=2(2 \pi)^{-2 s} \sqrt{\pi} \Gamma\left(s-a_{j}\right) \Gamma\left(s-b_{j}\right) D_{F, j}(s) .
$$

Here, $a_{j}$ and $b_{j}$ are determined by

$$
a_{j}, b_{j}=\frac{1 \pm \sqrt{1+4 \mu_{j}}}{4}
$$

where $\Delta \overline{v_{j}}=\mu_{j} \overline{v_{j}}$. Since $\Phi_{F, j}(s)$ is an entire function of $s([\mathrm{Im}]$, p. 929) we conclude that $D_{F, j}(s)$ does not vanish identically.

Now let $\varphi_{\lambda}=E_{\bar{u}}$ with $u \in \mathbb{C}$, Re $u=\frac{1}{2}$. By the results of [Im] for Re $s \gg 0$ we have

$$
\Phi_{F, u}(s)=2(2 \pi)^{-2 s} \sqrt{\pi} \Gamma\left(s-\frac{\bar{u}}{2}\right) \Gamma\left(s+\frac{\bar{u}-1}{2}\right) D_{F, u}(s),
$$

hence it follows that $D_{F, u}(s)$ does not vanish identically.

\section{$\S 3$. Andrianov identities}

Let again $F \in S_{k}\left(\Gamma_{2}\right)$ with Fourier coefficients $a(T)$. We assume that $F$ is an eigenform of all Hecke operators $T(m)(m \in \mathbb{N})$ with eigenvalues $\lambda_{F}(m)$.

According to Andrianov ([An], p. $84 \mathrm{f}$.), for any discriminant $D<0$ we have the identity

(3) $L_{D}(s-k+2, \chi) \sum_{i=1}^{h(D)} \chi\left(N_{i}\right) \sum_{m=1}^{\infty} \frac{a\left(m N_{i}\right)}{m^{s}}=\psi_{F}^{D}(s, \chi) Z_{F}(s) \quad(\operatorname{Re} s \gg 0)$.

Here $D=d f^{2}$ with $d$ fundamental and $H(D)=H\left(\mathcal{O}_{f}\right)$ is the group of classes of similar modules for the coefficient ring $\mathcal{O}_{f}$ with discriminant $D$ in the quadratic field $\mathbb{Q}(\sqrt{d})$. Furthermore, $N_{1}, \ldots, N_{h(D)}$ is a set of representatives of such modules, $\chi$ is a character of $H(D)$ and $L_{D}(s, \chi)$ denotes the $L$-function of $\mathcal{O}_{f}$ twisted with the character $\chi$. 
Note that we have a bijection $M \mapsto \widetilde{M}$ between $H(D)$ and the $\operatorname{SL}(2 ; \mathbb{Z})$ classes of half-integral, positive definite, primitive matrices with discriminant $D$. Putting $\widetilde{a}(m ; M):=a(m \widetilde{M})$ for $m \in \mathbb{N}$, the function $\psi_{F}^{D}(s, \chi)$ is defined by

$$
\psi_{F}^{D}(s, \chi)=\sum_{\delta_{1}|\delta| f} \frac{\mu(\delta) \mu\left(\delta_{1}\right)}{\delta^{s-k+2} \delta_{1}^{s-2 k+3}} \sum_{i=1}^{h(D)} \chi\left(N_{i}\right) \widetilde{a}\left(\frac{\delta}{\delta_{1}} ; \mathcal{O}_{\frac{f}{\delta}} N_{i}\right),
$$

where $\mathcal{O}_{\frac{f}{\delta}}$ is the order of discriminant $d\left(\frac{f}{\delta}\right)^{2}$ in $\mathbb{Q}(\sqrt{d})$ and $\mu$ denotes the Möbius function. Furthermore,

$$
Z_{F}(s)=\zeta(2 s-2 k+4) \sum_{m=1}^{\infty} \frac{\lambda_{F}(m)}{m^{s}} \quad(\operatorname{Re} s \gg 0)
$$

is the spinor zeta function attached to the eigenform $F$.

Inverting the character sum on the left-hand side of (3) we find that for each $j=1, \ldots, h(D)$ we have

$$
\sum_{m=1}^{\infty} \frac{a\left(m N_{j}\right)}{m^{s}}=\frac{1}{h(D)}\left(\sum_{\chi} \bar{\chi}\left(N_{j}\right) \frac{\psi_{F}^{D}(s, \chi)}{L_{D}(s-k+2, \chi)}\right) Z_{F}(s),
$$

where the sum is taken over all characters $\chi$ of $H(D)$.

From (5) we can deduce for $\operatorname{Re} s \gg 0$ an identity between $D_{F, \lambda}(s)$ $\left(\lambda \in \Lambda_{k}\right)$ and the spinor zeta function, namely

$$
\begin{aligned}
D_{F, \lambda}(s) & =\sum_{T>0} \sum_{\text {primitive } / \sim}^{\infty} \frac{a(m T) \varphi_{\lambda}\left(\frac{m}{\sqrt{\operatorname{det} m T}} T\right)}{\epsilon(m T)(\operatorname{det} m T)^{s}} \\
& =\sum_{T>0 \text { primitive } / \sim} \frac{\varphi_{\lambda}\left(\frac{1}{\sqrt{\operatorname{det} T}} T\right)}{\epsilon(T)(\operatorname{det} T)^{s}} \sum_{m=1}^{\infty} \frac{a(m T)}{m^{2 s}} \\
& =\frac{1}{2} \sum_{D \text { Diskr. }} \frac{4^{s}}{|D|^{s}} \sum_{i=1}^{h(D)} \frac{\varphi_{\lambda}\left(\frac{1}{\sqrt{\operatorname{det} N_{i}}} N_{i}\right)}{\epsilon\left(N_{i}\right)} \sum_{m=1}^{\infty} \frac{a\left(m N_{i}\right)}{m^{2 s}} \\
& =\frac{1}{2} K_{F, \lambda}(s) Z_{F}(2 s)
\end{aligned}
$$


where

$$
K_{F, \lambda}(s)=\sum_{\substack{D \text { Diskr. } \\ D<0}} \frac{4^{s}}{h(D)|D|^{s}} \sum_{i=1}^{h(D)} \frac{\varphi_{\lambda}\left(\frac{1}{\sqrt{\operatorname{det} N_{i}}} N_{i}\right)}{\epsilon\left(N_{i}\right)} \sum_{\chi} \bar{\chi}\left(N_{i}\right) \frac{\psi_{F}^{D}(2 s, \chi)}{L_{D}(2 s-k+2, \chi)} .
$$

\section{$\S 4$. Proof of Theorem}

Let $F \in S_{k}\left(\Gamma_{2}\right)$ be an eigenform of all Hecke operators $T(m)(m \in \mathbb{N})$. Given $\lambda \in \Lambda_{k}$, the corresponding function

$$
s \mapsto \Phi_{F, \lambda}(s)
$$

(notations as in $§ 2$ ) has a meromorphic continuation to the whole complex plane and satisfies the functional equation

$$
\Phi_{F, \lambda}(k-s)=(-1)^{k} \Phi_{F, \lambda}(s)
$$

([Im], p. 929).

On the other hand, by $[\mathrm{An}]$, p. 88,

$$
Z_{F}^{*}(s):=(2 \pi)^{-2 s} \Gamma(s) \Gamma(s-k+2) Z_{F}(s)
$$

has a meromorphic continuation to the whole complex plane and satisfies the functional equation

$$
Z_{F}^{*}(2 k-2-s)=(-1)^{k} Z_{F}^{*}(s)
$$

Proposition 4.1. Let $F, G \in S_{k}\left(\Gamma_{2}\right)$ be eigenforms of all Hecke operators $T(m)(m \in \mathbb{N})$ with Fourier coefficients $a(T)$ resp. $b(T)$.

If $a(n T)=b(n T)$ for every half-integral, positive definite, primitive matrix $T$ and every square-free integer $n \in \mathbb{N}$, then

$$
K_{F, \lambda}(s)=K_{G, \lambda}(s) \quad(\operatorname{Re} s \gg 0) .
$$

for any $\lambda \in \Lambda_{k}$.

Proof. We only have to check that $\psi_{F}^{D}(s, \chi)=\psi_{G}^{D}(s, \chi)$ for any discriminant $D<0$ and any character $\chi$ of $H(D)$. In the definition (4) we 
may consider $\mathcal{O}_{\frac{f}{\delta}} N_{i}$ as an element of $H\left(d\left(\frac{f}{\delta}\right)^{2}\right)([\mathrm{An}]$, p. 72). Substituting $m:=\frac{\delta}{\delta_{1}}$ we must sum over all $m|\delta| f$, and due to the factor $\mu(\delta)$ only square-free values of $m$ give a non-zero contribution. By assumption, for such values $m$ we have

$$
\widetilde{a}\left(m ; \mathcal{O}_{\frac{f}{\delta}} M_{i}\right)=a\left(m \widetilde{\mathcal{O}_{\frac{f}{\delta}} M_{i}}\right)=b\left(m \widetilde{\mathcal{O}_{\frac{f}{\delta}}} M_{i}\right)=\widetilde{b}\left(m ; \mathcal{O}_{\frac{f}{\delta}} M_{i}\right),
$$

which proves what we wanted.

Now let $F, G$ be as in Proposition 4.1 and $\lambda \in \Lambda_{k}$.

First suppose that $D_{F, \lambda}(s)$ is not identically zero. By (6) and Proposition 4.1 the same holds for $D_{G, \lambda}(s)$ and we have

$$
C(s):=\frac{D_{F, \lambda}(s)}{D_{G, \lambda}(s)}=\frac{Z_{F}(2 s)}{Z_{G}(2 s)} \quad(s \in \mathbb{C}),
$$

hence also

$$
C(s)=\frac{\Phi_{F, \lambda}(s)}{\Phi_{G, \lambda}(s)}=\frac{Z_{F}^{*}(2 s)}{Z_{G}^{*}(2 s)} \quad(s \in \mathbb{C}) .
$$

By (7)

$$
C(k-s)=C(s)
$$

and by (8)

$$
C(s)=C(k-1-s)
$$

Together we obtain

$$
C(s)=C(k-s)=C(k-1-(s-1))=C(s-1),
$$

i.e. $C$ is periodic modulo 1 . Since $Z_{G}(2 s)$ has no zeroes in some half plane Re $s \gg 0$ due to the convergent Euler product we conclude that $C(s)$ is holomorphic in this region, hence due to the periodicity, $C(s)$ is an entire function.

Clearly there exist $c>0$ and $K_{1}, K_{2}>0$ such that

$$
K_{1}<\left|Z_{F}(2 s)\right|,\left|Z_{G}(2 s)\right|<K_{2}
$$

for any $s \in \mathbb{C}$ with $c \leq \operatorname{Re} \mathrm{s} \leq c+1$. Therefore

$$
|C(s)|=\frac{\left|Z_{F}(2 s)\right|}{\left|Z_{G}(2 s)\right|} \leq \frac{K_{2}}{K_{1}}
$$


for such $s$ and hence for all $s \in \mathbb{C}$. Thus $C(s)$ is constant, hence $C(s)=1$. Therefore, $D_{F, \lambda}(s)=D_{G, \lambda}(s)$.

On the other hand, if $D_{F, \lambda}(s)$ is identically zero, then by (6) and Proposition 4.1 also $D_{G, \lambda}(s)$ is zero.

Hence, $D_{F, \lambda}(s)=D_{G, \lambda}(s)$ for every $\lambda \in \Lambda_{k}$ which by Lemma 2.1 implies $F=G$ (alternatively, for $k$ even one also could use [Ki]).

\section{REFERENCES}

[An] Andrianov, A.N., Euler products corresponding to Siegel modular forms of genus 2, Russ. Math. Surv., 29:3 (1974), 45-116, from: Uspekhi Mat. Nauk. 29:3 (1974), 43-110.

[Im] Imai, K., Generalization of Hecke's correspondence to Siegel modular forms, Amer. J. Math., 102, No. 5 (1980), 903-936.

[Ki] Kitaoka, Y., Representations of quadratic forms and their application to Selberg's zeta function, Nagoya Math. J., 63 (1976), 153-162.

[Ko] Kohnen, W., On Hecke eigenforms of half-integral weight, Math. Ann., 293 (1992), 427-431.

[Ku] Kubota, T., Elementary Theory of Eisenstein series, Halsted Press, 1973.

[Za] Zagier, D.B., Zetafunktionen und quadratische Körper, Springer-Verlag, Berlin, Heidelberg, New York, 1981.

Stefan Breulmann

Universität Heidelberg

Mathematisches Institut

Im Neuenheimer Feld 288

D-69120 Heidelberg

Germany

stef an . breulmann@urz. uni-heidelberg.de

Winfried Kohnen

Universität Heidelberg

Mathematisches Institut

Im Neuenheimer Feld 288

D-69120 Heidelberg

Germany

winfried@mathi.uni-heidelberg.de 